\title{
Bystander suppression of occupational hapten sensitization in rats made tolerant to ovalbumin
}

\author{
T. Pullerits*, S. Lundin**, Z-H. Cui*, U. Dahlgren**, E. Telemo**, J. Lötvall*
}

Bystander suppression of occupational hapten sensitization in rats made tolerant to ovalbumin. T. Pullerits, S. Lundin, Z-H. Cui, U. Dahlgren, E. Telemo, J. Lötvall. OERS Journals Ltd 1998.

ABSTRACT: Feeding a soluble antigen to an animal is known to cause a state of unresponsiveness against this antigen. If this antigen is given together with another antigen during the sensitization procedure, impairment of the response to the new antigen can also be seen, a phenomenon referred to as bystander suppression. The induction of tolerance against ovalbumin (OvA) and the effect of bystander suppression on the response to the hapten trimellitic anhydride (TMA), a cause of occupational asthma, were studied in Brown-Norway rats.

Rats were fed either OvA-containing pellets or standard diet for 16 days before sensitization with the mixture of TMA and OvA. The animals were followed for 6 weeks after sensitization.

Animals made tolerant to OvA showed a significantly suppressed delayed-type hypersensitivity (DTH) reaction against both OvA and TMA compared with the nontolerized control group at 5 weeks after sensitization, implying bystander suppression. By contrast, immunoglobulin (Ig)E and IgG antibody levels were suppressed only against OvA, whereas anti-TMA antibody levels were not affected. Airway eosinophilia after a single aerosol challenge at 6 weeks after sensitization using TMA conjugated to rat serum albumin, correlated with IgE anti-TMA levels in the group made tolerant to OvA and was not affected by OvA ingestion.

In conclusion, suppressive factors released in ovalbumin-tolerant rats when they are challenged with ovalbumin, can suppress the response to trimellitic anhydride and this suppression is more pronounced for T-helper1-type responses. Eur Respir J 1998; 12: 889-894.
*Lung Pharmacology Group, Dept of Respiratory Medicine and Allergology and **Dept of Clinical Immunology, Göteborg University, Gothenburg, Sweden.

Correspondence: T. Pullerits

Lung Pharmacology Group

Guldhedsgatan $10 \mathrm{~A}$

S-413 46 Gothenburg

Sweden

Fax: 4631413290

Keywords: Airway eosinophilia

bystander suppression

delayed-type hypersensitivity

immunoglobulin E

immunoglobulin G

oral tolerance

Received: November 201997

Accepted after revision June 291998

This study was supported by the Swedish Heart and Lung Foundation, the Swedish Work Environmental Foundation, the Swedish Medical Research Council and the Vårdal Foundation.
Oral administration of a soluble protein leads to immunological tolerance to this antigen $[1,2]$. This can be demonstrated experimentally by feeding a protein antigen and testing the immune response against the same antigen following parenteral sensitization [3]. Suggested mechanisms to explain antigen-driven tolerance include clonal deletion $[4,5]$, clonal anergy $[6,7]$ and active suppression [8-10]. In the last case, suppressor cells can be triggered through an antigen-specific mechanism, but their effect may act in a nonspecific manner by the release of certain cytokines such as interleukin (IL)-10 or transforming growth factor$\beta$ (TGF- $\beta$ ) $[8,11]$. Such production of antigen nonspecific suppressive cytokines can also result in diminished responses to an unrelated antigen in the close vicinity, known as bystander suppression [12]. Bystander suppression has been demonstrated in several autoimmune disease models, including suppression of experimental allergic encephalomyelitis by feeding myelin basic protein $[11,13]$ or ovalbumin [12], suppression of antigen-induced arthritis by type II collagen $[14,15]$ and suppression of virus-induced diabetes by insulin [16]. In a rat model of focal cerebral ischaemia, tolerance to myelin basic protein decreased stroke size [17]. Initial human trials using oral administration of antigen have shown positive results in patients with multiple sclerosis and rheumatoid arthritis, and a phase III trial of oral myelin in multiple sclerosis patients as well as phase II trials of orally administered type II collagen in rheumatoid arthritis, S-antigen in uveitis and insulin in type I diabetes are in progress [18]. Theoretically, bystander suppression can be a promising approach to treat allergic diseases, especially if the disease-inducing antigen is not known or several antigens are synergizing in the pathogenesis of the disease.

Trimellitic anhydride (TMA) is a hapten, capable of inducing occupational asthma [19]. Animal models using TMA have shown increased levels of immunoglobulin (Ig) $\mathrm{E}$ and IgG anti-TMA antibodies after sensitization [20] as well as increased airflow obstruction, plasma exudation and eosinophilic inflammation in the airways after antigen challenge $[21,22]$. In studies evaluating bystander suppression in animals made tolerant by ingestion of antigen, the bystander antigen has been a protein in most cases [10, $12,23]$. The availability of a clinically relevant and reproducible model of asthma, using a hapten antigen, prompted the investigation of whether bystander suppression affects the response to the hapten TMA. It was hypothesized that bystander suppression has the capacity to attenuate the response to the hapten and that such attenuation is also accompanied by reduced airway eosinophilia after aerosolized antigen challenge. 


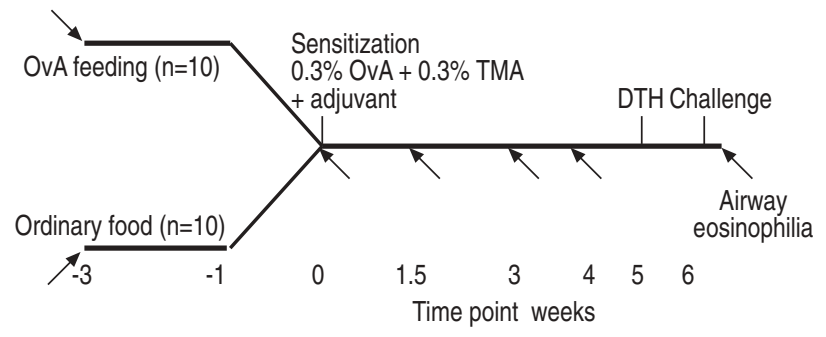

Fig. 1. - Experimental design of the study. Arrows indicate time points of taking serum samples. OvA: ovalbumin; TMA: trimellitic anhydride; DTH: delayed-type hypersensitivity.

\section{Materials and methods}

\section{Animals and study design}

Experimental design of the study is shown in figure 1. Twenty 7-8 week old male Brown Norway rats (Harlan UK, Oxon, UK), weighing 150-195 g, were divided into two study groups ( $\mathrm{n}=10$ in both groups). To induce tolerance to ovalbumin (OvA), one group was fed OvA-containing pellets (AnalyCen, Lidköping, Sweden), in which half of the standard protein content had been replaced with equal parts of egg protein and milk whey protein [24], for 16 days, a period used effectively for induction of oral tolerance in other studies [8]. The control group received standard diet for the same period. From day 17 all rats were given a standard diet. Six days after the removal of the OvA-pellets ( 3 weeks after the beginning of the study), all rats were sensitized with a mixture of $300 \mu \mathrm{g}$ of OvA and $300 \mu \mathrm{g}$ of TMA in $100 \mu \mathrm{L}$ of corn-oil and $100 \mu \mathrm{L}$ of Freund's complete adjuvant (FCA), given as two intradermal injections $24 \mathrm{~h}$ apart on each side of the back of the animal. The rats were then followed for 6 weeks, during which period repeated blood samples were taken and delayed-type hypersensitivity (DTH) was evaluated as described below. Nine weeks after the beginning of the study (6 weeks after sensitization) rats were challenged with $0.3 \%$ of aerosolized TMA conjugated to rat serum albumin (TMARSA) for 15 min in a chamber. Aerosol was generated by a nebulizer (Maxin MA2; Clinova Medical AB, Malmö, Sweden). Twenty-four hours after the challenge final blood samples were collected and lungs were removed from the thoracic cavity and fixed in formalin for making histology samples.

\section{Delayed-type hypersensitivity}

DTH response was evaluated 5 weeks after sensitization, because high levels of anti-TMA antibodies have previously been detected at that time point [20]. The DTH response to OvA and TMA was evaluated by intradermal injection of $20 \mu \mathrm{L}$ of $0.3 \%$ of OvA in phosphate-buffered saline (PBS) in one ear and $20 \mu \mathrm{L}$ of $0.3 \%$ of TMA in olive oil in the other ear. The ear thickness was measured in a blinded fashion before and $24 \mathrm{~h}$ after injection, using a micrometre calliper (Oditest; Kröplin, Hessen, Germany). The DTH response was expressed as the increase in ear thickness.
Enzyme-linked immunosorbent assay for assessment of specific immunoglobulin $E$ and $G$ antibodies

Blood samples for the evaluation of $\operatorname{IgE}$ and $\mathrm{IgG}$ antibody levels were collected by tail bleeding before induction of tolerance, before the sensitization and 1.5, 3, 4 and 6 weeks after sensitization. To evaluate $\operatorname{IgE}$ and IgG antiOvA and anti-TMA antibody levels, microtitre plates (Corning Glass Works, Corning, NY, USA) were coated with 5 $\mu \mathrm{g} \cdot \mathrm{mL}^{-1}$ of OvA or TMA-RSA in PBS coating buffer (PBS with $0.05 \%$ Tween-80), covered with plastic wrap and incubated overnight at $4^{\circ} \mathrm{C}$ in a humid atmosphere. Serum samples in double dilutions starting from 1:20 for IgE and from 1:100 for IgG were then added to the plates and incubated at $4{ }^{\circ} \mathrm{C}$ overnight or at room temperature for $2 \mathrm{~h}$, respectively. Bound anti-OvA and anti-TMA antibodies were detected by incubating the plates with sheep anti-rat IgE antibodies (epsilon chain) (1:4,000; ICN Biomedicals, Aurora, Ohio, USA) or rabbit anti-rat IgG antibodies (1:10,000; Zymed Laboratories, San Francisco, CA, USA), followed by incubation with alkaline phosphatase-conjugated ligands, affinity-purified rabbit anti-sheep IgG antibodies $(1: 10,000 ; \mathrm{ICN})$ or goat anti-rabbit IgG antibodies (1:10,000, Sigma Chemical Co., St Louis, MO, USA), respectively. Finally, a chromogen $p$-nitrophenyl phosphate substrate (PNPP) (Sigma) dissolved in diethanolamine buffer $\mathrm{pH} 9.8$ (1.0 mg of PNPP in $1.0 \mathrm{~mL}$ buffer) was added to visualize the bound ligand and the absorbance was read at $405 \mathrm{~nm}$ using a spectrophotometer (Labsystems Multiscan Multisoft; Labsystems Oy, Helsinki, Finland). Between every incubation the plates were washed four times with PBS with $0.05 \%$ Tween- 80 . The antibody levels are expressed as arbitrary ELISA units calculated by comparing absorbance values of different dilutions of the test sera with a standard curve from hyperimmune rat serum using a computer program written by U. Dahlgren.

\section{Histology for airway eosinophilia}

Slides for histology were cut from formalin-fixed lungs and stained with haematoxylin and eosin. Eosinophils in the bronchial mucosa were counted under a light microscope (Olympus BH-2; Olympus Optical Co., Tokyo, Japan) at magnification $40 \times$ in 40 high-power field areas along the airway, from the lobar bronchi to the terminal bronchioli for each sample.

\section{Data analysis}

Results are presented as mean \pm SEM. The statistical comparisons between the groups were performed using the Mann-Whitney U-test. To evaluate the production of antiOvA and anti-TMA antibodies during the post-sensitization period, the area under the curve (AUC) from before sensitization until week-4 post-sensitization, was calculated by combining individual measured time-points. The latter time-point of week-4 was chosen to avoid interference from re-exposure to antigens during DTH reaction. Spearman rank correlation was used for evaluation of correlation between airway eosinophilia and IgE anti-TMA antibodies. A p-value $<0.05$ was considered to be significant. 

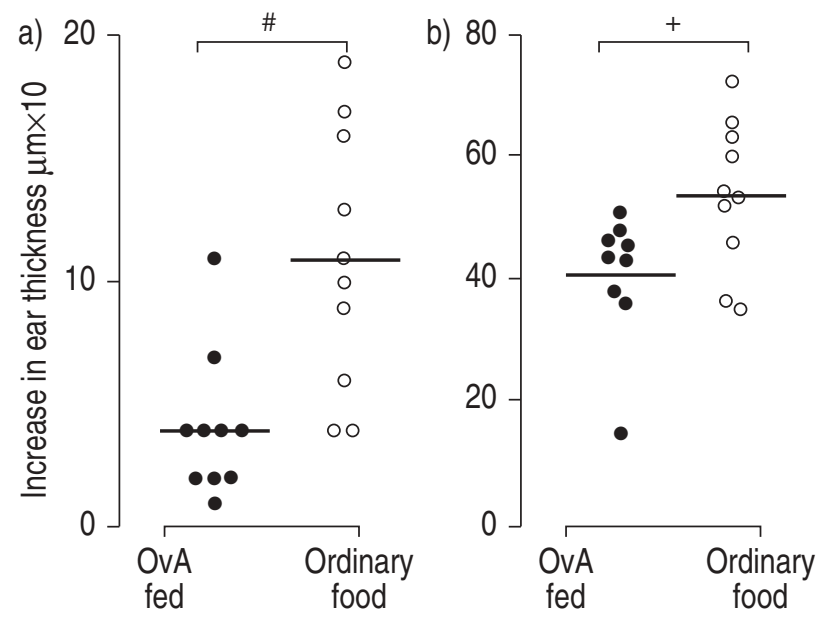

Fig. 2. - Delayed-type hypersensitivity reaction against a) ovalbumin (OvA) and b) trimellitic anhydride (TMA) measured as an increase in ear thickness 5 weeks after sensitization with a mixture of OvA and TMA. Each symbol represents one rat. — : indicates the mean of the group. ${ }^{+}$: $\mathrm{p}=0.03$; ${ }^{\#}$ : $\mathrm{p}=0.005$.

\section{Results}

\section{Delayed-type hypersensitivity}

The DTH reaction, evaluated $24 \mathrm{~h}$ after antigen injection, was significantly weaker in animals made tolerant to OvA than in control animals against both OvA (mean increase in ear thickness, expressed as $\mu \mathrm{m} \times 10,4.1 \pm 0.9$ versus $10.9 \pm$ 1.7, $\mathrm{p}=0.005)$ and TMA $(40.7 \pm 3.6$ versus $53.6 \pm 3.8$, $\mathrm{p}=0.03$ ) (fig. 2), indicating tolerance against OvA and bystander suppression of the T-helper (Th) 1 response against TMA in OvA-fed rats.

One rat from the group made tolerant to OvA was excluded from the analysis after TMA injection in the ear, because a marked haematoma developed at the injection site.

\section{Immunoglobulin $E$ and $G$ antibody levels}

Anti-OvA and anti-TMA antibodies were undetectable at baseline (figs. 3 and 4). After 16 days of OvA feeding

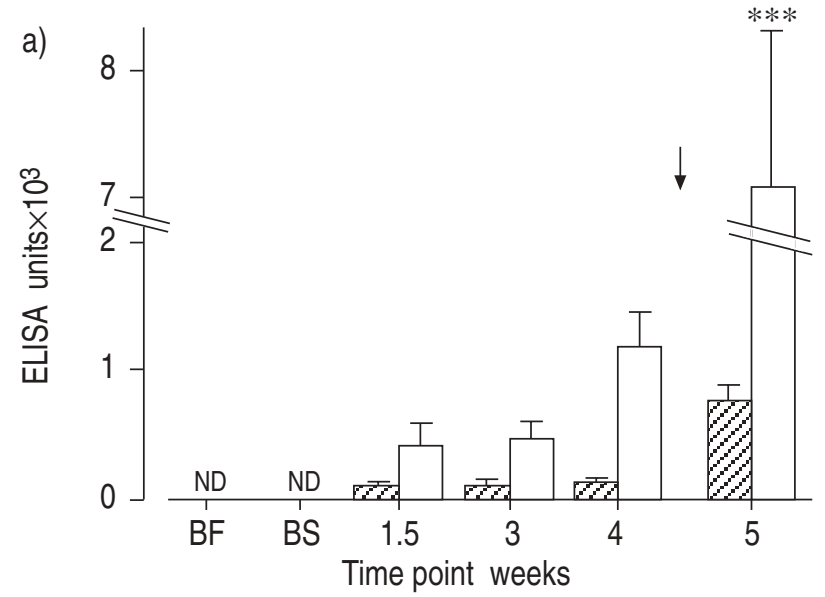

all OvA-fed animals produced low levels of IgG anti-OvA antibodies $(510 \pm 140$ enzyme-linked immunosorbent assay (ELISA) units), but not IgE anti-OvA antibodies. These IgG anti-OvA antibody levels were significantly higher than in the control group $(\mathrm{p}<0.001)$, in which levels of both IgG and IgE anti-OvA antibodies were undetectable (fig. 4).

Following sensitization with TMA and OvA, five out of 10 rats made tolerant to OvA and eight out of 10 control rats produced detectable levels of IgE anti-OvA antibodies 1.5 weeks after sensitization, while all rats produced detectable levels of IgE anti-TMA antibodies at the same time point (fig. 3). For statistical evaluation of antibody levels, the AUC from before sensitization until week 4 post-sensitization was calculated. The levels of $\operatorname{IgE}$ antiOvA antibodies were significantly lower in animals made

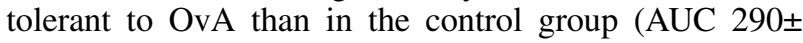
110 versus $1,800 \pm 430$ ELISA units.week, respectively, $p=$ 0.003 ), indicating tolerance against OvA in OvA-fed rats. However, the levels of IgE anti-TMA antibodies were not significantly different between the two study groups (AUC $2,410 \pm 390$ in rats made tolerant to OvA versus $1,640 \pm 230$ in the control group), implying that bystander suppression in rats made tolerant to OvA was not present for the Th2 response. DTH evaluation, performed at week 5 after sensitization, was followed by a sharp increase in IgE antibody levels in both groups. However, this challenge did not alter the established patterns of antibody levels. The final levels for IgE anti-OvA antibodies were $770 \pm 130$ ELISA units in OvA-tolerized versus 7,100 $\pm 1,250$ in the control group $(\mathrm{p}<0.001)$ and for IgE anti-TMA antibodies $33,500 \pm 8,050$ versus $18,800 \pm 4,750(\mathrm{p}=0.17)$, respectively.

Following sensitization with OvA and TMA all rats produced both IgG anti-OvA and anti-TMA antibodies, measurable from 1.5 weeks post-sensitization and onwards (fig. 4). During 4 weeks following sensitization the relative increase in IgG anti-OvA antibodies above presensitization levels was significantly lower in rats made tolerant to OvA than in the control group (AUC above presensitization levels 7,200 $\pm 1,710$ versus $17,300 \pm 4,370$ ELISA units.week, $\mathrm{p}=0.049$ ), although there were no differences in overall IgG anti-OvA levels between the two

b)

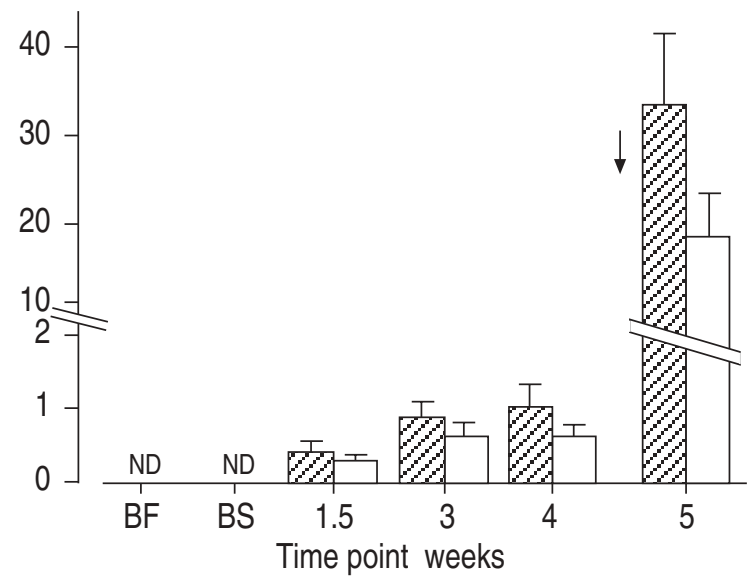

Fig. 3. - Immunoglobulin (Ig) E antibodies in serum against a) ovalbumin (OvA) and b) trimellitic anhydride (TMA) at different time points. $Z_{\text {ts }}$ made tolerant against OvA by ingestion before sensitization (BS); $\quad \square$ tolerant rats ( $\mathrm{n}=10$ in both groups). The area under the curve from before sensitization until week 4 after sensitization was significantly lower in rats made tolerant to OvA than in the control group for IgE anti-Ova antibodies $(\mathrm{p}=0.003)$, but not for IgE anti-TMA antibodies. The data are shown as means \pm SEM. ND: not detectable; ELISA: enzyme-linked immunosorbent assay; BF: before feeding. $\downarrow$ : delayed-type hypersensitivity at 5 weeks. $* * *: p<0.001$. 

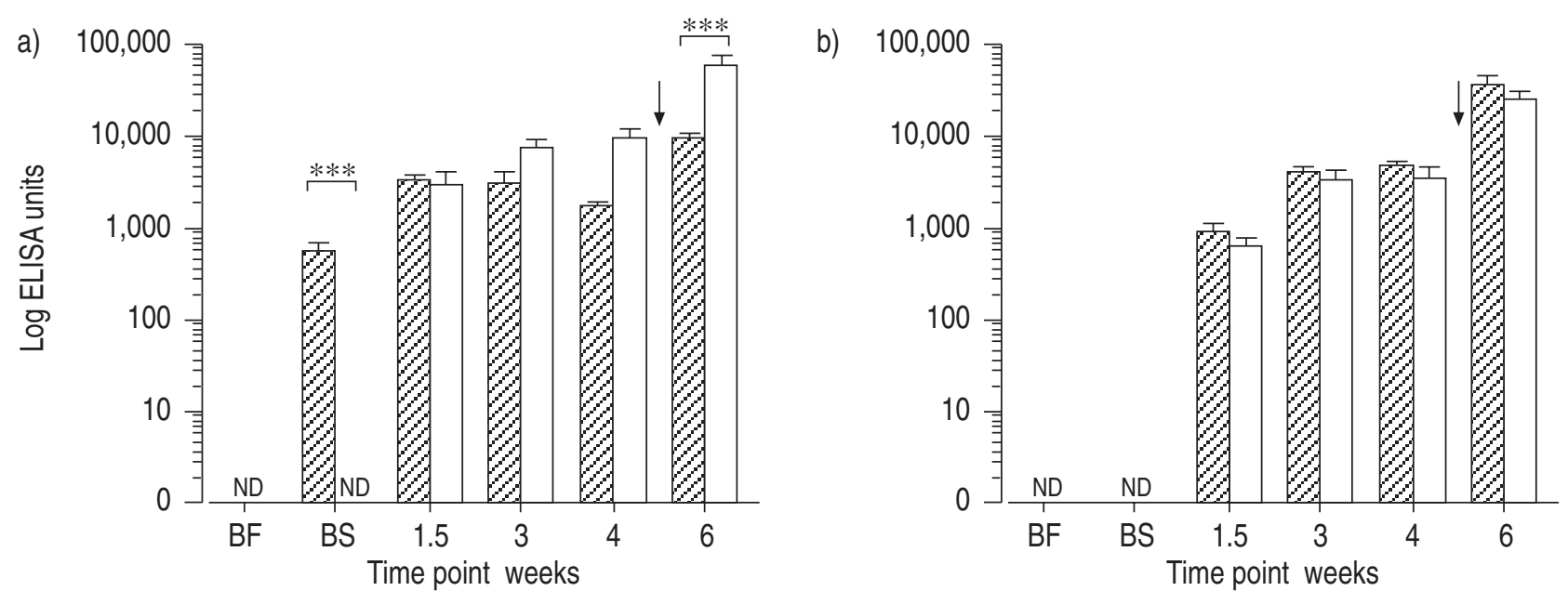

Fig. 4. - Immunoglobulin ( $\mathrm{Ig}) \mathrm{G}$ antibodies in serum against a) ovalbumin (OvA) and b) trimellitic anhydride (TMA) at different time points. $Z 2$ : rats made tolerant against OvA by ingestion before sensitization (BS); $\square$ : nontolerant rats (n=10 in both groups). After OvA feeding and before sensitization, OvA-fed rats had significantly higher levels of IgG anti-OvA antibodies than the control group. During 4 post-sensitization weeks the relative increase above presensitization antibody levels was significantly lower in rats made tolerant to OvA than in the control group for IgG anti-OvA antibodies (area under the curve; $\mathrm{p}<0.05$ ), but not for IgG anti-TMA antibodies. The data are shown as means \pm SEM. ND: not detectable; ELISA: enzymelinked immunosorbent assay; BF: before feeding. $\downarrow$ : delayed-type hypersensitivity at 5 weeks. ***: $\mathrm{p}<0.001$

groups (AUC for total levels of IgG anti-OvA antibodies $9,200 \pm 1,670$ versus $17,300 \pm 4,370, \mathrm{p}=0.13)$. The levels of IgG anti-TMA antibodies were not significantly different between the two study groups (AUC 9,600 950 versus $7,400 \pm 1,710$ ). This confirmed the presence of tolerance against OvA in OvA-fed rats but lack of bystander suppression against TMA for the Th2 response. Again, there was a sharp increase in the antibody levels after DTH evaluation, the final levels for IgG anti-OvA antibodies being $8,900 \pm 1,350$ ELISA units in rats made tolerant to OvA versus $55,500 \pm 11,200$ in the control group $(\mathrm{p}<0.001)$ and for IgG anti-TMA antibodies $39,100 \pm 10,100$ versus $28,400 \pm 4,470(\mathrm{p}=0.65)$, respectively.

\section{Airway eosinophilia}

There were no differences in the number of eosinophils in bronchial mucosa $24 \mathrm{~h}$ after challenge with TMA-RSA between the two groups $(74.1 \pm 21.1$ eosinophils per counted area in rats made tolerant to OvA versus $62.5 \pm 19.7$ eosinophils in the control rats). The airway eosinophilia correlated with the IgE anti-TMA antibodies, measured at the same time point, in the group made tolerant to OvA $(r=0.67, p=0.044)$, but not in the control group $(r=0.21)$.

\section{Discussion}

In the present study the presence of bystander suppression to the hapten allergen TMA was demonstrated in OvA-tolerant rats. Both the DTH reaction (marker of Th1 involvement) and the levels of $\operatorname{IgE}$ and $\mathrm{IgG}$ antibodies (Th2) were suppressed to OvA. However, the response to TMA was suppressed only for the DTH reaction, while the IgE and IgG anti-TMA antibodies and airway eosinophilia after a single allergen exposure were unaffected.

Previous studies demonstrating bystander suppression have, in most cases, used foreign proteins as bystander antigens $[10,12,23]$. The present study was able to show that bystander suppression also influences the response to a hapten, known to be immunogenic only upon binding to a protein carrier, in this case probably a self-protein. It has previously been shown that TMA induces an immune response after forming a link with a lysine residue of autologous proteins and that the antibody response in that case is directed against new antigenic determinants which are formed upon conjugation of the hapten with the protein [25]. Thus, the response to a hapten-modified self-protein is also affected by bystander suppression.

In animal models bystander suppression has been shown to attenuate both Th1 and Th2 responses, the latter generally being evaluated by suppressed IgE antibody levels [23]. The present authors wanted to assess the involvement of another Th2 marker, development of airway eosinophilia after allergen challenge. Possible attenuation of airway eosinophilia might have clinical importance, since some previous studies have found that airway eosinophilia is closely related to increased bronchial hyperreactivity [26], although this is not a constant finding [27]. However, infiltration of eosinophils into the airways $24 \mathrm{~h}$ after aerosol challenge with TMA-RSA was not attenuated in the group made tolerant to OvA in the present study. The data do not exclude that a simultaneous challenge with tolerance inducing and bystander antigens might give a different result, as for the development of eosinophilic inflammation in animals made tolerant by ingestion.

Previous studies using antigen feeding as means of generating peripheral oral tolerance have shown that tolerance preferably occurs on the Th1 level [28-30], while the Th2 response often appears to be intact. Similar difficulties in generating Th2 tolerance were seen in experimental models in which tolerance was induced by the i.p. administration of soluble antigen [31, 32]. It has been suggested that this is due to a rapid physiological clearance of proteins or protein fragments from the circulation by glomerular filtration [33], so that they cannot provide an adequate signal for the induction of Th2 tolerance; Th2 are known to be more resistant to tolerance than Th1 lymphocytes [34]. Thus, it has been shown that a single high dose of OvA induces 
Th1-tolerance [30], which can be extended to include Th2type responses by increasing the length of administration of soluble antigen to 20 days [35]. In the present study, suppression of IgE anti-OvA antibody production could be induced with 2 weeks of continuous feeding of high doses of OvA before sensitization. However, the same feeding regimen did not alter the development of $\operatorname{IgE}$ antibodies against the bystander TMA antigen, implying that it is even more difficult to induce Th2 suppression on a bystander level.

A critical point in induction of bystander suppression is the method of sensitization. Thus, it has been demonstrated that in rats, made tolerant to OvA by ingestion, the suppressed T- and B-cell response to an unrelated antigen, human serum albumin (HSA), was present only if sensitization was performed with a mixture of OvA and HSA. When rats were sensitized to OvA and HSA on two separate sites on the back of the animal, there was no dif-ference in the response to HSA in rats made tolerant by ingestion and nontolerant rats [23]. The latter finding also excluded the possibility of shared epitopes between the two antigens. The importance of the sensitization site was further stressed by MLLER et al. [12], demonstrating that bystander suppression can be induced even if the two antigens were injected $8 \mathrm{~h}$ apart, but at the same site. Thus, the same injection site for sensitization seems to be a more important determinant in the induction of bystander suppression than a similar time-point for sensitization. In the present protocol, the sensitization to OvA and TMA was performed using the mixture of these two antigens in the same syringe and this can, therefore, not account for the failure to induce bystander suppression of $\operatorname{IgE}$.

In summary, these data provide evidence that feeding a soluble antigen can induce tolerance to a bystander hapten antigen if these antigens are administered together during the sensitization and that this suppression preferably affects T-helper 1-type responses.

Acknowledgements: The authors thank B-E. Skoogh for valuable advice and discussion during the progress of this study.

\section{References}

1. Friedman A, al-Sabbagh A, Santos LM, et al. Oral tolerance: a biologically relevant pathway to generate peripheral tolerance against external and self antigens. Chem Immunol 1994; 58: 259-290.

2. Weiner HL. Oral tolerance: immune mechanisms and treatment of autoimmune diseases. Immunol Today 1997; 18: 335-343.

3. Chiller JM, Glasebrook AL. Oral tolerance and the induction of T cell unresponsiveness. Monogr Allergy 1988; 24: 256-265.

4. Jones LA, Chin LT, Longo DL, Kruisbeek AM. Peripheral clonal elimination of functional T cells. Science 1990; 250: 1726-1729.

5. Webb S, Morris C, Sprent J. Extrathymic tolerance of mature T cells: clonal elimination as a consequence of immunity. Cell 1990; 63: 1249-1256.

6. Ramsdell F, Lantz T, Fowlkes BJ. A nondeletional mechanism of thymic self tolerance. Science 1989; 246: 10381041.
7. Melamed D, Friedman A. Direct evidence for anergy in T lymphocytes tolerized by oral administration of ovalbumin. Eur J Immunol 1993; 23: 935-942.

8. Miller A, Lider O, Roberts AB, Sporn MB, Weiner HL. Suppressor T cells generated by oral tolerization to myelin basic protein suppress both in vitro and in vivo immune responses by the release of transforming growth factor beta after antigen-specific triggering. Proc Natl Acad Sci USA 1992; 89: 421-425.

9. Miller JF, Morahan G. Peripheral T cell tolerance. Annu Rev Immunol 1992; 10: 51-69.

10. Lundin BS, Dahlgren UI, Hanson LÅ, Telemo E. Oral tolerization leads to active suppression and bystander tolerance in adult rats while anergy dominates in young rats. Scand J Immunol 1996; 43: 56-63.

11. Chen Y, Kushroo VK, Inobe J, Hafler DA, Weiner HL. Regulatory $\mathrm{T}$ cell clones induced by oral tolerance: suppresion of autoimmune encephalomyelitis. Science 1994; 265: 1237-1240.

12. Miller A, Lider O, Weiner HL. Antigen-driven bystander suppression after oral administration of antigens. $J$ Exp Med 1991; 174: 791-798.

13. Al-Sabbagh A, Miller A, Santos LMB, Weiner HL. Antigen-driven tissue-specific suppression following oral tolerance: orally administered myelin basic protein suppresses proteolipid protein-induced experimental autoimmune encephalomyelitis in the SJL mouse. Eur J Immunol 1994; 24: 2104-2109.

14. Zhang ZJ, Lee CSY, Lider O, Weiner HL. Suppression of adjuvant arthritis in Lewis rats by oral administration of type II collagen. J Immunol 1990; 145: 2489-2493.

15. Yoshino S, Quattrocchi E, Weiner HL. Suppression of antigen-induced arthritis in Lewis rats by oral administration of type II collagen. Arthritis Rheum 1995; 38: 1092-1096.

16. von Herrath MG, Dyrberg T, Oldstone MBA. Oral insulin treatment suppresses virus-induced antigen-specific destruction of beta cells and prevents autoimmune diabetes in transgenic mice. J Clin Invest 1996; 98: 1324-1331.

17. Becker KJ, McCarron RM, Ruetzler C, et al. Immunologic tolerance to myelin basic protein decreases stroke size after transient focal cerebral ischemia. Proc Natl Acad Sci USA 1997; 94: 10873-10878.

18. Weiner HL. Oral tolerance for the treatment of autoimmune diseases. Annu Rev Med 1997; 48: 341-351.

19. Venables KM. Low molecular chemicals, hypersensitivity, and direct toxicity: the acid anhydrides. Br J Ind Med 1989; 46: 222-232.

20. Pullerits T, Dahlgren U, Skoogh BE, Lötvall J. Development of antigen-specific IgE after sensitisation with trimellitic anhydride in rats is attenuated by glucocorticoids and cyclosporin A. Int Arch Allergy Immunol 1997; 112: 279286.

21. Arakawa H, Lötvall J, Kawikova I, et al. Airway allergy to trimellitic anhydride in guinea pigs: different time courses of IgG1 titer and airway responses to allergen challenge. J Allergy Clin Immunol 1993; 92: 425-434.

22. Cui ZH, Sjöstrand M, Pullerits T, Andius P, Skoogh BE, Lötvall J. Bronchial hyperresponsiveness, epithelial damage, and airway eosinophilia after single and repeated allergen exposure in a rat model of anhydride-induced asthma. Allergy 1997; 52: 739-746.

23. Dahlman-Höglund A, Dahlgren U, Ahlstedt S, Hanson $\mathrm{LA}$, Telemo E. Bystander suppression of the immune response to human serum albumin in rats fed ovalbumin. Immunology 1995; 86: 128-133.

24. Wold AE, Dahlgren UI, Hanson LÅ, Mattsby-Baltzer I, Midvetdt T. Difference between bacterial and food antigens 
in mucosal immunogenicity. Infect Immun 1989; 57: 26662673.

25. Zeiss CR, Levitz D, Chacon R, Wolkonsky P, Patterson R, Pruzansky JJ. Quantitation and new antigenic determinant specificity of antibodies induced by inhalation of trimellitic anhydride in man. Int Arch Allergy Appl Immunol 1980; 61: 380-388.

26. Elwood W, Barnes PJ, Chung KF. Airway hyperresponsiveness is associated with inflammatory cell infiltration in allergic brown-Norway rats. Int Arch Allergy Immunol 1992; 99: 91-97.

27. Kips JC, Cuvelier CA, Pauwels RA. Effect of acute and chronic antigen inhalation on airway morphology and responsiveness in actively sensitized rats. Am Rev Respir Dis 1992; 145: 1306-1310.

28. Friedman A, Weiner HL. Induction of anergy or active suppression following oral tolerance is determined by antigen dosage. Proc Natl Acad Sci USA 1994; 91: 6688-6692.

29. Fishman-Lobell J, Friedman A, Weiner HL. Different kinetic patterns of cytokine gene expression in vivo in orally tolerant mice. Eur J Immunol 1994; 24: 2720-2724.
30. Melamed D, Friedman A. In vivo tolerization of Th1 lymphocytes following a single feeding with ovalbumin: anergy in the absence of suppression. Eur J Immunol 1994; 24: 1974-1981.

31. Burstein HJ, Shea CM, Abbas AK. Aqueous antigens induce in vivo tolerance selectively in IL-2- and IFN-gammaproducing (Th1) cells. J Immunol 1992; 148: 3687-3691.

32. De Wit D, Van Mechelen M, Ryelandt M, et al. The injection of deaggregated gamma globulins in adult mice induces antigen-specific unresponsiveness of T helper type 1 but not type 2 lymphocytes. J Exp Med 1992; 175: 9-14.

33. Romball CG, Weigle WO. In vivo induction of tolerance in murine CD4+ cell subsets. J Exp Med 1993; 178: 16371644.

34. Peterson JD, Karpus WJ, Clatch RJ, Miller SD. Split tolerance of Th1 and Th2 cells in tolerance to Theiler's murine encephalomyelitis virus. Eur J Immunol 1993; 23: 46-55.

35. Melamed D, Fishman-Lovell J, Uni Z, Weiner HL, Friedman A. Peripheral tolerance of Th2 lymphocytes induced by continuous feeding of ovalbumin. Int Immunol 1996; 8: 717-724. 